\title{
In the absence of Lgt, lipoproteins are shed from Streptococcus uberis independently of Lsp
}

\author{
Correspondence \\ J. A. Leigh \\ james.leigh@nottingham.ac.uk
}

Received 11 July 2008

Revised 5 September 2008

Accepted 10 September 2008
E. L. Denham, ${ }^{1}$ P. N. Ward ${ }^{2}$ and J. A. Leigh ${ }^{3}$

\author{
${ }^{1}$ Institute for Animal Health, Compton, Berkshire RG20 7NN, UK \\ ${ }^{2}$ Nuffield Department of Clinical Laboratory Sciences, Oxford University, John Radcliffe Hospital, \\ Headington, Oxfordshire OX3 9DU, UK \\ ${ }^{3}$ School of Veterinary Medicine and Science, University of Nottingham, Sutton Bonington, \\ Leicestershire LE12 5RD, UK
}

\begin{abstract}
The role of lipoprotein diacylglyceryl transferase (Lgt) and lipoprotein signal peptidase (Lsp) responsible for processing lipoproteins was investigated in Streptococcus uberis, a common cause of bovine mastitis. In the absence of Lgt, three lipoproteins [MtuA (SUB0473), Hap (SUB1625) and an extracellular solute-binding protein (SUB0365)] were detected in extracellular locations. All were shown by Edman degradation analysis to be cleaved on the carboxy side of the LXXC lipobox. Detection of MtuA, a lipoprotein shown previously to be essential for infectivity and virulence, was used as a surrogate lipoprotein marker to locate and assess processing of lipoproteins. The absence of Lgt did not prevent location of MtuA to the cell membrane, its location in the wild-type strain but, in contrast to the situation with wild-type, did result in a widespread location of this protein. In the absence of both Lgt and Lsp, MtuA was similarly released from the bacterial cell. In such strains, however, the cell-associated MtuA represented the full-length gene product, indicating that Lsp was able to cleave non-lipidated (lipo)proteins but was not responsible for their release from this bacterium.
\end{abstract}

\section{INTRODUCTION}

Lipoproteins are a major class of membrane-bound proteins that were first identified in Escherichia coli, but have since been shown in many pathogenic bacteria including Staphylococcus aureus, Mycobacterium tuberculosis and streptococcal species (Hamilton et al., 2006; Leskela et al., 1999; Petit et al., 2001; Stoll et al., 2005; Sutcliffe \& Harrington, 2004; Sutcliffe \& Russell, 1995). Lipoproteins perform critical roles within bacteria; these include facilitating nutrient uptake (Janulczyk et al., 2003), mediating antibiotic resistance (Sutcliffe \& Russell, 1995), protein folding and the processing of extracytoplasmic proteins emerging from the cell (Kontinen et al., 1991; Wahlstrom et al., 2003). In Streptococcus uberis, a common cause of bovine mastitis worldwide, the lipoprotein MtuA was shown to be essential for active transport of $\mathrm{Mn}^{2+}$, growth in milk and virulence in the target species (Smith et al., 2003).

Lipoproteins are synthesized with an $\mathrm{N}$-terminal signal sequence that directs the protein into the lipoproteinprocessing pathway. The lipobox motif (LXXC) contained within the C-terminal region of the signal peptide (Sutcliffe \& Harrington, 2002; von Heijne, 1989) acts as a target for lipoprotein diacylglyceryl transferase (Lgt). This enzyme effects lipid modification and consequent anchoring of the lipoprotein to the cytoplasmic membrane (Hantke \&
Braun, 1973; Leskela et al., 1999). In a number of bacteria, it has been demonstrated that lipid modification of the cysteine residue of the lipobox is a prerequisite for cleavage of the signal peptide by the lipoprotein signal peptidase (Lsp) (Hussain et al., 1982; Tjalsma et al., 1999a; Tokunaga $\& \mathrm{Wu}, 1984)$.

In a study using the signal peptide region of Braun's lipoprotein from E. coli, it was revealed that Lgt used phosphatidylglycerol to anchor the protein to the membrane while other, similar, molecules such as phosphatidylethanolamine or cardiolipin were not suitable substrates for this enzyme (Sankaran \& Wu, 1994). Lgt transfers the diacylglycerol moiety from phosphatidylglycerol to the thiol group of the cysteine residue with the formation of $\mathrm{N}$ acyl-S-diacylglyceryl cysteine. Once lipidation has occurred, Lsp recognizes the modified cysteine residue and cleaves the signal peptide sequence, leaving the lipidmodified cysteine residue as the new $\mathrm{N}$ terminus of the protein (Hussain et al., 1982).

Lgt is ubiquitous across bacterial species. The biosynthetic anchoring pathway of lipoproteins in Gram-negative bacteria has been elucidated in considerable detail (Braun \& Hantke, 1975; Chattopadhyay \& Wu, 1977; Dev \& Ray, 1984; Hantke \& Braun, 1973; Hussain et al., 1982; Innis et al., 1984; Inukai et al., 1984; Lee et al., 1983; Tokunaga \& Wu, 1984; Tokunaga et al., 1982, 1985; Yamagata, 1983; 
Yamagata et al., 1982; Yamaguchi et al., 1988). In Grampositive bacteria, Lgt was first reported in Staph. aureus, where the gene encoding Lgt was identified by complementation of a temperature-sensitive defect in an lgt mutant of E. coli (Qi et al., 1995). A high level of similarity was found between the amino acid sequences of Lgt from E. coli, Salmonella typhimurium, Haemophilus influenzae and Staph. aureus (Qi et al., 1995).

The functional role of Lgt has also been studied in Bacillus subtilis (Antelmann et al., 2001; Leskela et al., 1999), where, unlike the situation in Gram-negative species, a mutant lacking Lgt remained fully viable (Gan et al., 1993; Leskela et al., 1999; Yamagata et al., 1982). A major lipoprotein of B. subtilis, PrsA, was shown to be larger in the absence of Lgt activity, suggesting the presence of an intact signal peptide sequence. These findings provided evidence that the pathway for lipoprotein processing in some Grampositive species of bacteria may be similar to that detected in Gram-negative bacteria, in that lipidation was a prerequisite for removal of the signal peptide by Lsp.

In B. subtilis, lipoproteins have been shown to be shaved from the cell surface by cleavage on the carboxy side of the LXXC motif (Antelmann et al., 2001). In Staph. aureus, the absence of Lgt resulted in the release of several (lipo)proteins (SitC, PrsA and OppA) (Stoll et al., 2005). In each case, the released proteins were processed to a size similar to that of the mature, fully processed (lipo)protein, whereas cell-associated lipoproteins expressed in the absence of Lgt appeared to retain their signal sequence. In Listeria monocytogenes, the absence of Lgt resulted in the release of mature (lipo)proteins from the cell. In this strain, treatment with the Lsp inhibitor globomycin prevented both (lipo)protein cleavage and release, implying that Lsp was able to cleave non-lipidated (lipo)proteins and that such cleavage was a prerequisite for their release.

Lgt homologues are present in all streptococcal species sequenced to date, and lipoproteins represent an abundant group of streptococcal proteins with a wide range of functions (Sutcliffe \& Russell, 1995). Interestingly, disruption of Lgt-prelipoprotein processing appears to influence the localization of lipoproteins and consequent pathogenicity of Gram-positive pathogens in a variety of ways (Henneke et al., 2008). A Streptococcus pneumoniae lgt mutant was shown to be viable, but exhibited reduced virulence (Petit et al., 2001). The pathogenicity of an lgt mutant in Streptococcus equi was reduced but not eliminated, indicating that it is not an absolute requirement for virulence in a challenge model in the horse (Hamilton et al., 2006). The lipoprotein MtuA from Strep. uberis was previously shown to be essential for disease within dairy cattle (Smith et al., 2003) and provided a useful marker with which to help dissect the role of Lgt in the pathogenesis of Strep. uberis.

This communication describes the use of isogenic mutants lacking Lgt and, for the first time, Lsp and Lgt to elucidate the processing of lipoprotein. In so doing, it adds to the understanding of lipoprotein processing in Gram-positive bacteria.

\section{METHODS}

Bacterial growth conditions and oligonucleotide primers. Strep. uberis was routinely grown on Todd-Hewitt agar or sheep blood aesculin agar (SEA) plates and in Todd-Hewitt broth (THB) (Oxoid) as standing cultures at $37{ }^{\circ} \mathrm{C}$. Uncured $\mathrm{pGh} 9^{+}:$: ISS1 mutants were grown in the same media with the addition of $1 \mu \mathrm{g}$ erythromycin $\mathrm{ml}^{-1}$ at $37^{\circ} \mathrm{C}$. Globomycin was added to cultures at an $\mathrm{OD}_{600}$ of 0.1 . Oligonucleotide primers P348 (5'-AGGACGAAATGTTTCAGTTG) and P349 (5'-AGATAACCAGTGCCACAAAG) were used to amplify the lgt locus and P432 (5'-GCTCTTCGGATTTTCGGTATC) and P571 (5'-AATATCTTCAGCTTCATAATCC) were used to amplify the lsp locus of Strep. uberis. Oligonucleotide primers P358 (5'CATTTTCCACGAATAGAAGGACTGTC) and P247 (5'GCTCTTCGGATTTTCGGTATC) were used to screen pGh9:: ISS1 mutant banks, as described below, and a DIG-labelled ISS1 probe was used for Southern blotting as described previously (Ward et al., 2001).

Preparation of chromosomal DNA from Strep. uberis. Chromosomal DNA from $3 \mathrm{ml}$ culture was prepared by using a variation of the method of Hill \& Leigh (1989) and is described in Denham et al. (2008).

Southern blotting to determine location and random distribution of pGh9' $^{+}$: ISS1 insertion. Southern blot analysis was performed upon genomic DNA digested with EcoRI or HindIII. Hybridization of the digoxigenin-dUTP (DIG-dUTP; Roche Diagnostics) labelled probe was carried out overnight at $65{ }^{\circ} \mathrm{C}$. Hybridizing fragments were visualized with the chemiluminescent substrate NBT/BCIP (Roche Diagnostics) as instructed by the manufacturer.

Isolation of mutants from a Strep. uberis strain 0140J pGhost9::ISS1 mutant bank. The mutants were isolated as described in Denham et al. (2008) using the specific oligonucleotide primers described earlier.

Random mutagenesis in Isp mutant background of Strep. uberis. The procedures described previously by Maguin et al. (1996) and Smith et al. (2003) were used to generate a bank of random mutants within the lsp mutant of Strep. uberis using the pGh9:: ISS1 vector. Mutants from this bank were isolated in the same manner as described earlier for the isolation of mutants from the Strep. uberis strain 0140J pGh9:: ISS1 mutant bank.

Detection of MtuA with whole-cell ELISA. Bacteria were grown to mid-exponential phase and the suspensions were adjusted to an $\mathrm{OD}_{550}$ of 0.3 with growth medium (THB). Suspensions were mixed with equal volumes of coating buffer (carbonate buffer $0.05 \mathrm{M}$, $\mathrm{pH}$ 9.6) and applied in triplicate to the wells of a 96-well MaxiSorp plate (Nunc). Antigen-negative controls contained only coating buffer in the wells. The plate was incubated overnight in a humid environment at $4{ }^{\circ} \mathrm{C}$ to promote binding. Wells were washed three times with ELISA wash buffer [PBS containing $0.1 \%(w / v)$ Tween; PBST] and blocking solution [PBST containing $1 \%(\mathrm{w} / \mathrm{v})$ Marvel] was added $(200 \mu \mathrm{l}$ per well) and the plate was then incubated for $90 \mathrm{~min}$ at room temperature. Wells were washed again prior to addition of MtuA antisera (diluted 1 in 1000 in blocking solution, $100 \mu \mathrm{l}$ per well) and incubated for $90 \mathrm{~min}$ at room temperature. Wells were washed as described above to remove unbound antibody and then incubated for $1 \mathrm{~h}$ with anti-rabbit HRP conjugate antibody (Sigma) (diluted 1 in 1000 in blocking solution). Antibodies were detected 
using TMB reagent $[0.1 \mathrm{ml}$ TMB dissolved in DMSO and $0.03 \%(\mathrm{v} / \mathrm{v})$ $\mathrm{H}_{2} \mathrm{O}_{2}$ in $10 \mathrm{ml}$ TMB substrate buffer $(0.1 \mathrm{M}$ sodium acetate, $\mathrm{pH} 6.0$ ), $100 \mu \mathrm{l}$ per well]. After approximately $3 \mathrm{~min}, 1 \mathrm{M} \mathrm{H}_{2} \mathrm{SO}_{4}$ was added $(100 \mu \mathrm{l}$ per well) to stop further colour generation. Absorbance readings were taken at $450 \mathrm{~nm}$ (Anthos 2001 plate reader; Anthos Labtec Instruments).

Preparation of cleared whole-cell lysates and subcellular fractions. Whole-cell lysates and subcellular fractions (capsule, cell wall, cell membrane and cell contents) were prepared as described previously (Denham et al., 2008).

Preparation of proteins from bacterial growth media by TCA precipitation. Bacteria were grown to an $\mathrm{OD}_{550}$ of approximately 0.5 . Protease inhibitors were added to a $1 \times$ concentration from a $25 \times$ stock of Complete EDTA-free protease inhibitor (Roche Diagnostics). Bacteria were harvested by centrifugation $(10000 \mathrm{~g}, 10 \mathrm{~min})$. The culture supernatants (approx. $45 \mathrm{ml}$ ) were transferred to a fresh centrifuge vessel before being centrifuged as described previously to remove residual bacteria. The culture supernatants were filtered through a $0.22 \mu \mathrm{m}$ filter into a fresh centrifuge vessel and placed on ice. Trichloroacetic acid (TCA) was added (10\% v/v from a $100 \%$ $\mathrm{w} / \mathrm{v}$ stock solution) and mixed thoroughly. Proteins were precipitated on ice for $30 \mathrm{~min}$ before being harvested by centrifugation $\left(13000 \mathrm{~g}, 30 \mathrm{~min}, 4{ }^{\circ} \mathrm{C}\right)$. The supernatant was discarded and the precipitate was washed with $5 \mathrm{ml}$ ice-cold acetone. The precipitate was recovered by centrifugation as described previously, before being washed and spun again to remove traces of TCA. The remaining precipitate was air-dried thoroughly before being resuspended in either SDS-PAGE loading buffer $\left(\mathrm{pH} \mathrm{8.0)}\right.$ or Tris/ $\mathrm{HCl}\left(\mathrm{pH} \mathrm{8.0)}\right.$ and frozen at $-20{ }^{\circ} \mathrm{C}$.

Quantification of protein within cell fractions. The protein content of cell fractions was determined using the BCA Protein assay kit (Perbio) as directed by the manufacturer. Protein concentrations were calculated from mean values of triplicate readings for each sample using a standard curve prepared using BSA.

Detection of MtuA within cell fractions prepared from Strep. uberis. The proteins present in cell fractions $(1 \mu \mathrm{g})$ were separated by SDS-PAGE on $12.5 \%$ gels or $10 \%$ Bistris gels (Invitrogen) and detected by Western blotting using MtuA antiserum (Jones et al., 2004) at a concentration of $1: 2500$ and a secondary goat anti-rabbitHRP conjugate (Sigma) at a concentration of $1: 2500$.

\section{RESULTS}

\section{The Igt locus in the Strep. uberis strain 0140J genome}

A gene (SUB0578) corresponding to lgt was identified within the genome of Strep. uberis strain 0140J by similarity searches (BLASTN; Altschul et al., 1990) with functionally characterized lgt sequences from B. subtilis (GenBank accession no. AAC67287; amino acid sequence identity $46 \%)$, E. coli (NP417305; $30 \%)$ and Strep. pneumoniae (YP_816710; 65\%). The ORF encoding Lgt in Strep. uberis was predicted to generate a product of 259 amino acids and, as in Staph. aureus (Stoll et al., 2005), was flanked upstream by an ORF (SUB0577) with homology to $h p r K$ [an ATP-dependent, metabolite-activated protein kinase/ phosphorylase (Reizer et al., 1998)] and downstream by an ORF (SUB0579) encoding an unknown hypothetical protein (data not shown).

\section{Isolation of mutants carrying lesions within the lgt locus of Strep. uberis strain 0140J}

Mutants carrying insertions in lgt were isolated using a genotypic selection procedure from a bank of approximately 8800 random insertion mutants (Ward et al., 2001). Three mutants were identified with insertions in the region of the lgt locus. Sequence analysis revealed that in two mutants the insertion mapped within SUB0578 (Strep. uberis lgt:: ISS $1_{1}$ had an insertion $416 \mathrm{bp}$ from the start codon and Strep. uberis lgt:: ISS1 $1_{2}$ had an insertion 729 bp from the start codon). A further mutant (lgt::ISS1 $\left.1_{3}\right)$ carried an insertion that mapped outside the ORF, $13 \mathrm{bp}$ after the stop codon. The insertion in Strep. uberis lgt::ISS1 1 was predicted to disrupt the Lgt signature sequence (PS01311) described in Prosite (Hulo et al., 2006).

\section{Characterization of the extracellular protein profile of the lgt mutants}

To determine whether a mutation in the lgt gene of Strep. uberis had any effect on the extracellular protein profile, capsule fractions were prepared from mid-exponential phase cultures of the wild-type strain and the three mutants (lgt::ISS1 $1_{1}, \operatorname{lgt}:: \mathrm{ISS1}_{2}$ and lgt::ISS1 $1_{3}$ ). Several proteins that were seen in capsule extracts from $\operatorname{lgt}:: \mathrm{ISS} 1_{1}$ were not evident in the wild-type strain, lgt::ISS1 $1_{2}$ or lgt:: $\mathrm{ISS}_{3}$ (Fig. 1). These proteins were immobilized on PVDF membrane and subjected to Edman degradation analysis. Three proteins, including MtuA, were identified (Table 1). These corresponded to predicted lipoproteins described by Denham et al. (2008). The N-terminal residue of each protein corresponded to the amino acid carboxyl to the cysteine residue of the lipobox (LXXC) motif, a site distinct from that predicted for the cleavage of lipoproteins by Lsp.

\section{Cell-surface display of MtuA on wild-type and Igt mutants}

Whole-cell ELISA was able to detect MtuA at similar, low levels on the wild-type, lgt:: $\mathrm{ISS}_{2}$ and $\operatorname{lgt}:: \mathrm{ISS}_{3}$. Mutant lgt:: ISS $1_{1}$ displayed a considerably higher level of this protein on its surface (Fig. 2). This observation, coupled with the different extracytoplasmic protein profile shown for lgt::ISS1 $1_{1}$ (Fig. 1), suggested that lgt::ISS1 $1_{1}$, unlike the wild-type, was unable to anchor lipoproteins conventionally to the membrane. Furthermore, the similarity between the findings for $\operatorname{lgt}:: \mathrm{ISS}_{2}$, lgt::ISS1 $1_{3}$ and the wild-type suggested that the phenotype observed for lgt::ISS1 $1_{1}$ was not due to polar effects on ORFs located downstream of lgt.

\section{The (lipo)protein MtuA is found in all cell fractions from Igt : : ISS1,}

Immunoblotting of cell fractions with anti-MtuA revealed a single protein of approximately $37 \mathrm{kDa}$ in the membrane 


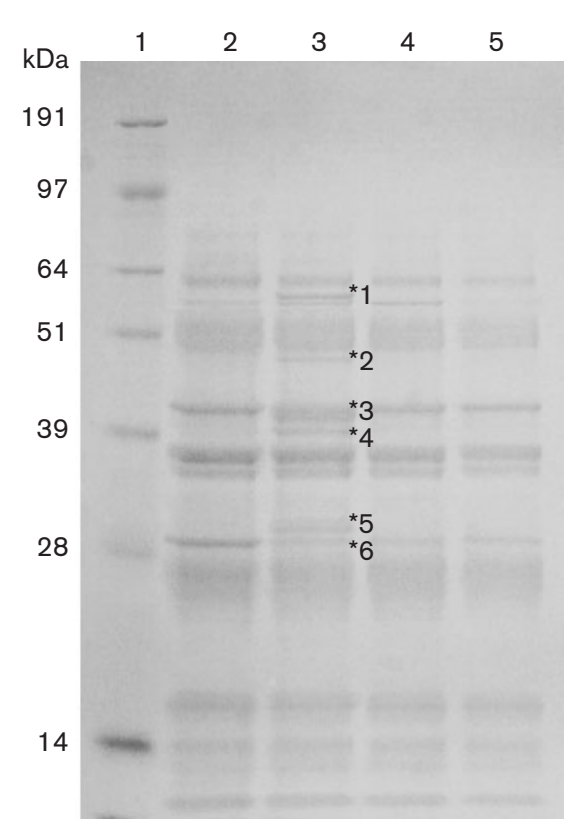

Fig. 1. SDS-PAGE protein profiles of capsule preparations from wild-type and Lgt mutant Strep. uberis. Capsule fractions were prepared from mid-exponential phase cultures of wild-type (lane 2) and three lgt mutants (ISS1::lgt $t_{1-3}$ ) (lanes 3-5). Lane 1, molecular mass marker proteins with sizes indicated in $\mathrm{kDa}$. Proteins were separated by SDS-PAGE and stained with Simply Blue SafeStain reagent (Invitrogen). At least six additional protein bands were detected in Igt::ISS1 $1_{1}$ (marked with asterisks 1-6). Three of these were identified by $\mathrm{N}$-terminal sequencing as hyaluronate-associated protein SUB1625 (*2), manganese transporter uberis (MtuA) SUB0473 (*4) and an extracellular solutebinding protein SUB0365 $\left({ }^{*} 6\right)$.

fraction of the wild-type strain (as shown previously; Jones et al., 2004). In contrast, strain lgt::ISS1 1 displayed a similar-sized protein in the capsule, cell wall, membrane, cytoplasm and culture supernatant fractions (Fig. 3).

\section{Processing of MtuA is carried out by Lsp in the absence of Lgt, but release of (lipo)proteins occurs independently of Lsp}

The three additional (lipo)proteins detected in the extracellular fraction of lgt:: ISS1 $1_{1}$ (Fig. 1) were atypically

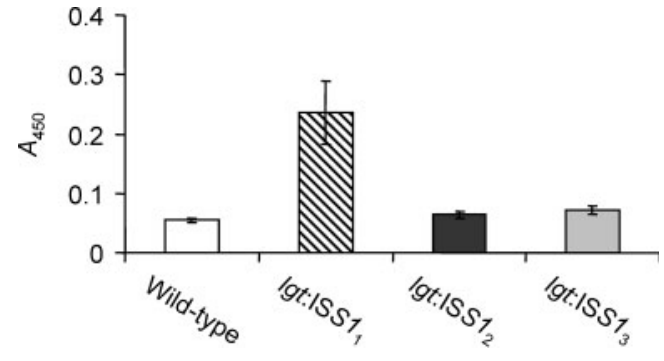

Fig. 2. Detection of MtuA on wild-type and lgt mutants by wholecell ELISA. Bacteria were grown to mid-exponential phase and the suspensions were adjusted to $\mathrm{OD}_{550} 0.3$ with THB. Anti-MtuA (Jones et al., 2004) was used as primary antibody to detect the presence of MtuA on the surface of whole cells. MtuA was found on the surface of $\operatorname{lgt}:: \mathrm{ISS} 1_{1}$ cells only. Error bars represent SD from assays performed in triplicate.

processed, as cleavage of the signal peptide was shown to have occurred on the carboxyl side of the cysteine residue of the lipobox motif. This suggested the presence of an activity other than Lsp that was able to cleave the signal peptides from (lipo)proteins.

Immunoblotting of whole-cell lysates of lgt:: $\mathrm{ISS}_{1}$ prepared from cultures growing exponentially in the presence of globomycin (an inhibitor of Lsp) revealed an additional MtuA protein at a size comparable to the full-length product of $m t u A$ (Fig. 4), indicating that Lsp was involved directly or indirectly in cleavage of unlipidated MtuA. In order to substantiate this further, an $l g t / l s p$ double mutant with an ISS1 insertion within lgt (546 bp from the start codon) was isolated from a random mutant bank created within a Strep. uberis 0140J lsp::ISS1 background (Denham et al., 2008) and characterized with regard to its lipoprotein-processing properties.

Immunoblotting of cell-associated fractions (cell wall, membrane and cytoplasm) from a mid-exponential phase culture of the lgt/lsp double mutant revealed an additional MtuA protein band of a molecular mass comparable to the full-length, uncleaved protein, as shown previously in the presence of this mutation within lsp (Denham et al., 2008) (Fig. 5a). However, immunoblotting of extracellular fractions [capsule (Fig. 5a) and culture supernatant (Fig. $5 b)$ ] revealed a single protein band comparable in size to

Table 1. Sequence of proteins identified by $\mathrm{N}$-terminal sequencing

Lipobox residues are shown in bold and the $\mathrm{N}$-terminal sequence derived from $\operatorname{lgt}:: \mathrm{ISS}_{1}$ is underlined.

\begin{tabular}{|ll|}
\hline ORF assignment & \multicolumn{1}{c|}{ Predicted lipoprotein leader sequence } \\
\hline SUB1625. Putative threonine kinase (hyaluronate-associated & MTVAQKSTFKRFGLGAVTLASAALLMAC GNKTAAKND \\
protein) or OppA1 (Sutcliffe \& Harrington, 2002) & MKKKLSLAIMAFLGLLMLGAC SVGNGRKAT \\
SUB0473. MtuA & MMTLKKNLGILSLTLGTLAILAAC GNKASNNSG \\
SUB0365. Putative bacterial extracellular solute-binding protein & \\
\hline
\end{tabular}


(a)

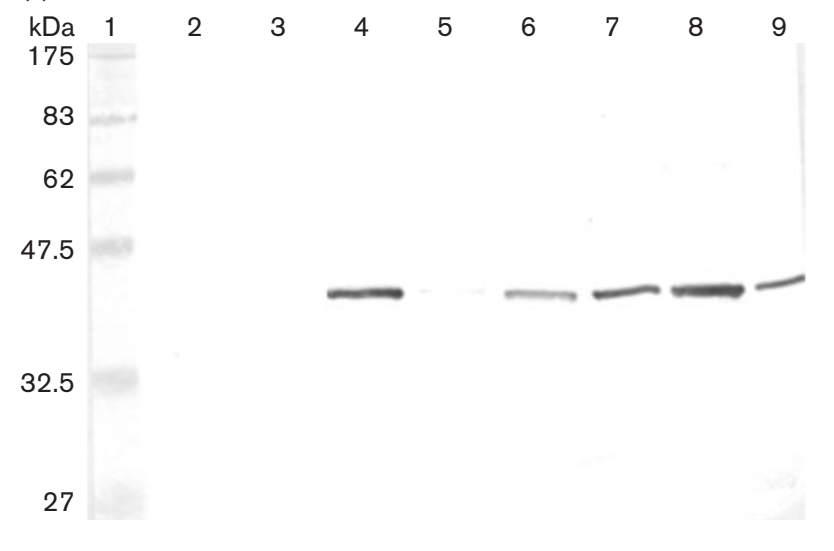

(b)

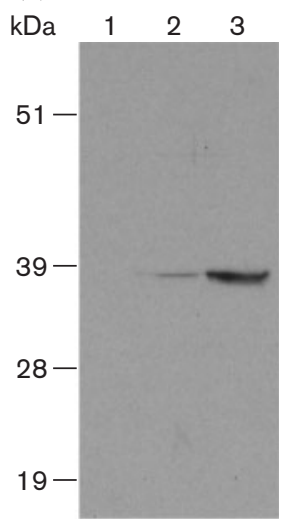

Fig. 3. Immunoblots showing the presence of MtuA within different subcellular fractions prepared from wild-type and the lgt mutant strain. (a) Lanes: 1, molecular mass marker (kDa); 2-5, wild-type capsule, cell wall, membrane and cell contents, respectively; 6 9, lgt mutant capsule, cell wall, membrane and cell contents, respectively. (b) Immunoblot of proteins prepared from the supernatant of midexponential phase Strep. uberis wild-type (lane 1) and lgt mutant (lane 2). Lane 3 is a membrane fraction prepared from the wildtype. Molecular masses of markers are shown in $\mathrm{kDa}$.
MtuA in all fractions from the single lgt mutant, indicating that MtuA was released in the absence of Lsp and that the released protein was cleaved similarly to that released in the presence of Lsp.

\section{DISCUSSION}

Lipid modification of the cysteine residue present within the lipobox of lipoproteins by Lgt has been shown to be essential for anchoring such proteins to the membrane of bacteria (Tjalsma et al., 1999a; Tokunaga \& Wu, 1984). In Gram-negative bacteria, mutations in lgt are lethal, while in Gram-positive bacteria, mutation of lgt has resulted in viable bacterial strains displaying variously altered phenotypes (Baumgartner et al., 2007; Hamilton et al., 2006; Petit et al., 2001; Stoll et al., 2005). Lgt in Strep. uberis was predicted to have seven transmembrane domains. The insertion in lgt:: ISS $1_{1}$ maps immediately after the fourth

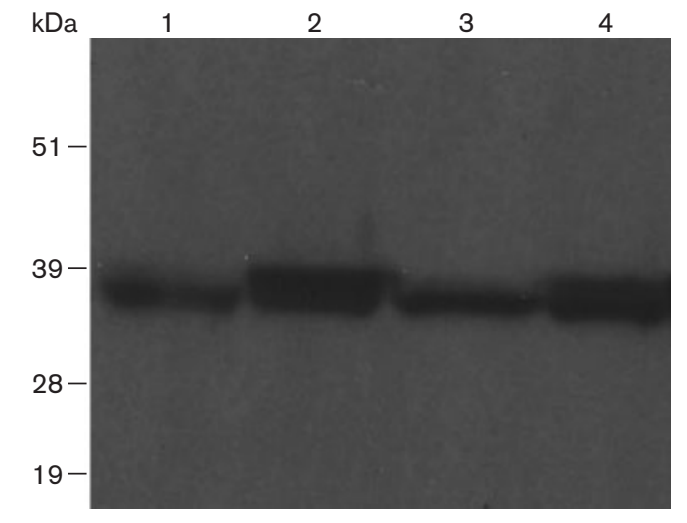

Fig. 4. Immunoblot of whole-cell lysates from mid-exponential phase cells. Samples were prepared from whole washed cells grown with/without globomycin, separated by SDS-PAGE and immunoblotted using anti-MtuA. Lanes: 1, wild-type; 2, wild-type plus globomycin; 3, lgt mutant; 4, lgt mutant plus globomycin. Molecular masses of markers are shown in $\mathrm{kDa}$. transmembrane domain and in the Prosite motif PS01311. The conserved amino acids within this motif are likely to play a role in either the catalysis of the diglyceride bond or the stability of the enzyme (Sankaran \& Wu, 1994). Therefore, any truncated Lgt from $\operatorname{lgt}: \mathrm{ISS}_{1}$ would not be predicted to be functional. The mutations in $l g t:: \mathrm{ISS}_{2}$ and lgt:: $\mathrm{ISS}_{3}$ mapped 729 bp from the start codon and $13 \mathrm{bp}$ after the stop codon, respectively. No difference was detected in the (lipo)protein distribution between lgt::ISS1 $1_{2}$ and the wild-type strain, indicating that disruption of lgt at this point has no major effect on the activity of Lgt. The last transmembrane domain is predicted to terminate at a sequence corresponding to $741 \mathrm{bp}$ from the start codon. A mutant strain of B. subtilis selected for defects in the KinB-dependent sporulation pathway (Dartois et al., 1997) was shown to carry a mutation in $l g t$ that was located at a position upstream of the lesion present in $\lg t:: \mathrm{ISS}_{2}$, suggesting a discrepancy between the essential regions of Lgt in these two bacteria.

Analysis of the proteins found within the capsule fractions from the three Strep. uberis lgt:: ISS 1 mutants revealed at least six additional proteins in lgt:: ISS1 1 that were not present either in the wild-type or in the other lgt mutants. Three of these were identified as putative lipoproteins and, in each case, the $\mathrm{N}$ terminus was shown to be the amino acid following the conserved cysteine of the lipobox. Lipid modification of cysteine within the lipobox motif by Lgt has been shown to be a prerequisite for the cleavage of the signal peptide from lipoproteins by Lsp in E. coli (Tokunaga \& Wu, 1984) and has been implied in Staph. aureus (Sibbald et al., 2006). However, cleavage of nonlipidated (lipo)proteins has been implied through the use of the selective inhibitor globomycin in L. monocytogenes (Baumgartner et al., 2007). Interestingly, in the extracellular proteome of wild-type B. subtilis, seven lipoproteins were shed into the extracellular medium and six of these were cleaved after LXXC (Antelmann et al., 2001) in a manner similar to those identified from $\operatorname{lgt}:: \mathrm{ISS} 1_{1}$ in the present study. The data presented here suggest the presence of an enzyme capable of shaving lipoproteins from the 
(a)

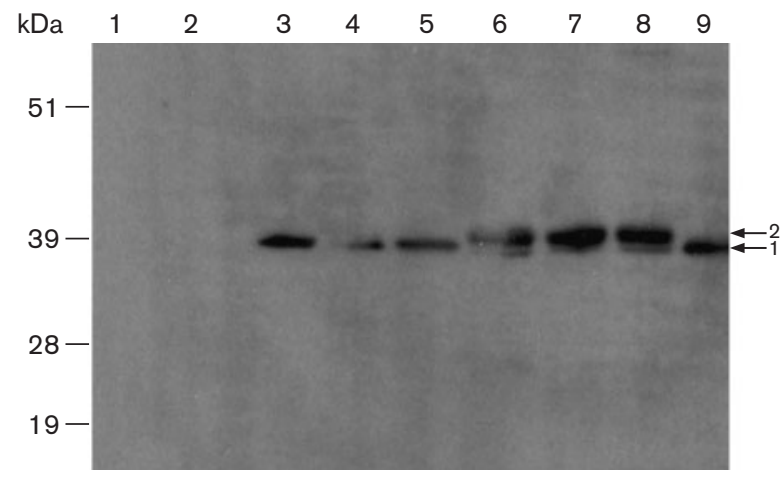

(b)

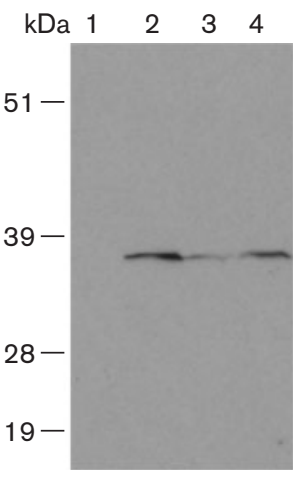

Fig. 5. Immunoblots of subcellular fractions of wild-type and the $/ g t / / s p$ double mutant using anti-MtuA. Samples were prepared using the subcellular fractionation procedure and immunoblotted using anti-MtuA to visualize differently processed forms of MtuA protein. (a) Lanes: 1-4, wild-type capsule, cell wall, membrane and cell contents, respectively; 58, lgt/lsp mutant capsule, cell wall, membrane and cell contents, respectively; 9, lgt capsule. Arrow 1 indicates the size of MtuA processed in the wild-type, lgt mutant and /gt/lsp mutants and arrow 2 indicates unprocessed MtuA detected in the /sp and /gt/lsp mutants. (b) Proteins from the supernatant prepared from mid-exponential-phase Strep. uberis wild-type (lane 1), lgt mutant (lane 2) and lgt/lsp mutant (lane 3). Membrane from the wild-type strain was included as a control (lane 4). MtuA was found in the supernatant of the two lipoproteinprocessing mutants. Molecular masses of markers are shown $(\mathrm{kDa})$. surface of Gram-positive bacteria, the activity of which is only apparent in Strep. uberis in the absence of Lgt.

In the absence of Lgt, MtuA was mislocated, and it could be envisaged that the non-lipidated protein might be positioned incorrectly with respect to accessory proteins (MtuB/C), thereby precluding active transport of $\mathrm{Mn}^{2+}$. Mutation within $m t u A / B / C$ resulted in a strain that was unable to grow in bovine milk, a medium in which $\mathrm{Mn}^{2+}$ is restricted (C. L. Jones and J. A. Leigh, unpublished). However, in the absence of Lgt, Strep. uberis was able to grow in milk at a rate and final cell yield similar to that of the wild-type, indicating that sufficient functional MtuA was present to acquire $\mathrm{Mn}^{2+}$ at a rate not limiting to growth. Mutation of the Strep. pneumoniae lgt gene produced bacteria that were 5 -fold reduced in their ability to cause infection (Petit et al., 2001). An lgt mutant and a maturase lipoprotein mutant have both been studied in Strep. equi, where it was found that the maturase mutant was less virulent than both the wild-type and lgt mutant (Hamilton et al., 2006). These data indicate that, while elimination of Lgt can constrain the pathogenesis of some Gram-positive pathogens, mutation of essential lipoproteins processed by Lgt can have a greater effect on the virulence of the pathogen. However, lgt mutants of Staph. aureus (Bubeck Wardenburg et al., 2006) and Streptococcus agalactiae (Henneke et al., 2008) were both hypervirulent within animal infection models through escaping detection by the host's innate immune system. Lipoproteins are therefore of high relevance to the host in mounting an effective immune responses to these two pathogens (Bubeck Wardenburg et al., 2006; Henneke et al., 2008). Contrastingly, while the lipoproteins of L. monocytogenes constitute immunological targets, an lgt mutant was found to be avirulent (Machata et al., 2008). A reduced Lgt mutant bacterial load within the spleen and liver was identified under these circumstances, suggesting the essential nature of Lgt-processed proteins in enabling the establishment of L. monocytogenes infections (Machata et al., 2008).

In contrast to the situation in the wild-type, in lgt:: ISS1, MtuA was detected in all fractions of the bacterium (cell contents, cell wall, cell membrane, capsule and culture supernatant). Full-length MtuA was not detected, demonstrating that MtuA [and probably other (lipo)proteins] was cleaved despite the absence of a lipid anchor. MtuA was detected in the cell contents of the lgt mutant in a processed form. It is important to note that the cellcontent fractions were prepared from disrupted protoplasts, i.e. a mixture of membrane and cytosolic contents. The lipoproteins in the Lgt mutants are not anchored and are therefore free to move into the cytoplasmic fraction (Figs 3 and 5a). The cytoplasmic cell fractions are likely to be enriched for proteins from that specific location and those loosely associated with the membrane, but it is highly unlikely that they will be contaminated by proteins from other locations, the fractions for which were removed prior to disruption of the protoplasts. In an $l s p$ mutant of Strep. uberis, alternative processing of MtuA was shown to be carried out by Eep (Denham et al., 2008). Eep activity was not detectable in the lgt mutant background reported in this study.

The localization of lipoproteins against backgrounds of $\lg t$ or $l s p$ mutations has been shown to be altered in other bacterial species including B. subtilis (Antelmann et al., 2001; Tjalsma et al., 1999a, b), Strep. agalactiae (Henneke 
et al., 2008), L. monocytogenes (Baumgartner et al., 2007) and Staph. aureus (Bubeck Wardenburg et al., 2006; Stoll et al., 2005). Lipoproteins in wild-type bacteria are in general anchored to the membrane. In B. subtilis and Staph. aureus, several lipoproteins have been found in the extracellular space as a result of alternative processing of the signal peptide (Antelmann et al., 2001; Sibbald et al., 2006). Inactivation of $l s p$ has not been shown to affect the location of lipoproteins. Furthermore, immunoblotting of cell fractions has shown that lipoproteins remain localized within the membrane under such circumstances, but alternatively processed forms of lipoproteins have been identified (Denham et al., 2008; Tjalsma et al., 1999a). These alternative forms are likely to contain varying amounts of the signal peptide. In Strep. uberis, full-length lipoprotein was detected along with an alternatively processed form, where Eep was shown to be responsible for the cleavage event at a site approximately 8 amino acids away from the Lsp cleavage site within the signal peptide (Denham et al., 2008). When lgt is inactivated, the location of at least some lipoproteins is altered. This alteration of location can vary for different lipoproteins in an $\mathrm{Lgt}^{-}$ background; for example, in Strep. agalactiae, the location of $\mathrm{Lmb}$ is the supernatant, whereas ScaA is released in part to the supernatant but also appears retained within the bacteria. This is also the case in an lgt/lsp double mutant (Henneke et al., 2008).

Evidence collected during analysis of extracts from $\operatorname{lgt}:$ : ISS $1_{1}$ grown in the presence of globomycin and from a strain of Strep. uberis 0140J lacking both Lsp and Lgt indicated that Lsp was able to remove the signal peptide from cellassociated (lipo)proteins in the absence of Lgt. The Nterminal sequence data from the lgt mutant suggested that the lipoproteins were processed on the C-terminal side of the cysteine residue of the lipobox. While it is conceivable that conventionally Lsp-cleaved forms of the proteins were also present in the capsule fraction, the relative abundance of the alternatively processed form was reflected in N-terminal sequence data. Cleavage of non-lipidated (lipo)proteins by Lsp of L. monocytogenes was implied by inhibition by globomycin (Baumgartner et al., 2007). However, in contrast to the situation in the present study with Strep. uberis, addition of globomycin to cultures of the lgt mutant of $L$. monocytogenes also prevented release of (lipo)proteins into the supernatant fraction. Consequently, it was hypothesized that the presence of a hydrophobic signal peptide (removed by Lsp and retained in the presence of globomycin) prevented the release of (lipo)proteins into the supernatant. This appeared not to be the case in our study of Strep. uberis, where the $l g t / l s p$ double mutant was capable of (lipo)protein release (Fig. 5b).

The data presented here show clearly that, in Strep. uberis, similar to other bacteria, Lgt and Lsp act to process lipoproteins; like the situation in L. monocytogenes, Lsp can cleave non-lipidated substrates, but, in contrast, Lsp from Strep. uberis is not required for release or shaving of such proteins from the bacterium. The observations on lipoprotein processing have indicated the pitfalls of extrapolating conclusions from one species to bacteria in general. The demonstration in E. coli that lipidation was a prerequisite for signal peptide cleavage has not proved to be universal. Similarly, the observation that Lsp acts as the release or shaving activity in $L$. monocytogenes does not appear to be true for Strep. uberis, where the shaving factor remains unidentified.

\section{ACKNOWLEDGEMENTS}

The authors acknowledge the financial support of the BBSRC (UK) for this research and are grateful to the Sanger Institute for supplying genomic sequence (http://www.sanger.ac.uk/Projects/S_uberis/) and annotation data (Matthew Holden, personal communication) prior to formal publication. Preliminary genomic sequence analysis was performed by Michael Watson and N-terminal sequence data were generated by Laurence Hunt, Institute for Animal Health. The authors also wish to thank Shunichi Miyakoshi, Sankyo Co., Japan, for the kind donation of globomycin.

\section{REFERENCES}

Altschul, S. F., Gish, W., Miller, W., Meyers, E. W. \& Lipman, D. J. (1990). Basic local alignment search tool. J Mol Biol 215, 403-410.

Antelmann, H., Tjalsma, H., Voigt, B., Ohlmeier, S., Bron, S., van Dijl, J. M. \& Hecker, M. (2001). A proteomic view on genome-based signal peptide predictions. Genome Res 11, 1484-1502.

Baumgartner, M., Karst, U., Gerstel, B., Loessner, M., Wehland, J. \& Jansch, L. (2007). Inactivation of Lgt allows systematic characterization of lipoproteins from Listeria monocytogenes. J Bacteriol 189, 313-324.

Braun, V. \& Hantke, K. (1975). Characterization of the free form of murein-lipoprotein from the outer membrane of Escherichia coli $\mathrm{B} / \mathrm{r}$. FEBS Lett 60, 26-28.

Bubeck Wardenburg, J., Williams, W. A. \& Missiakas, D. (2006). Host defenses against Staphylococcus aureus infection require recognition of bacterial lipoproteins. Proc Natl Acad Sci U S A 103, 13831-13836.

Chattopadhyay, P. K. \& Wu, H. C. (1977). Biosynthesis of the covalently linked diglyceride in murein lipoprotein of Escherichia coli. Proc Natl Acad Sci U S A 74, 5318-5322.

Dartois, V., Djavakhishvili, T. \& Hoch, J. A. (1997). KapB is a lipoprotein required for KinB signal transduction and activation of the phosphorelay to sporulation in Bacillus subtilis. Mol Microbiol 26, 1097-1108.

Denham, E. L., Ward, P. N. \& Leigh, J. A. (2008). Lipoprotein signal peptides are processed by Lsp and Eep of Streptococcus uberis. J Bacteriol 190, 4641-4647.

Dev, I. K. \& Ray, P. H. (1984). Rapid assay and purification of a unique signal peptidase that processes the prolipoprotein from Escherichia coli B. J Biol Chem 259, 11114-11120.

Gan, K., Gupta, S. D., Sankaran, K., Schmid, M. B. \& Wu, H. C. (1993). Isolation and characterization of a temperature-sensitive mutant of Salmonella typhimurium defective in prolipoprotein modification. $J$ Biol Chem 268, 16544-16550.

Hamilton, A., Robinson, C., Sutcliffe, I. C., Slater, J., Maskell, D. J., Davis-Poynter, N., Smith, K., Waller, A. \& Harrington, D. J. (2006). Mutation of the maturase lipoprotein attenuates the virulence of Streptococcus equi to a greater extent than does loss of general lipoprotein lipidation. Infect Immun 74, 6907-6919.

Hantke, K. \& Braun, V. (1973). Covalent binding of lipid to protein. Diglyceride and amide-linked fatty acid at the N-terminal end of the 
murein-lipoprotein of the Escherichia coli outer membrane. Eur J Biochem 34, 284-296.

Henneke, P., Dramsi, S., Mancuso, G., Chraibi, K., Pellegrini, E., Theilacker, C., Hubner, J., Santos-Sierra, S., Teti, G. \& other authors (2008). Lipoproteins are critical TLR2 activating toxins in group B streptococcal sepsis. J Immunol 180, 6149-6158.

Hill, A. W. \& Leigh, J. A. (1989). DNA fingerprinting of Streptococcus uberis: a useful tool for epidemiology of bovine mastitis. Epidemiol Infect 103, 165-171.

Hulo, N., Bairoch, A., Bulliard, V., Cerutti, L., De Castro, E., Langendijk-Genevaux, P. S., Pagni, M. \& Sigrist, C. J. (2006). The PROSITE database. Nucleic Acids Res 34, D227-D230.

Hussain, M., Ichihara, S. \& Mizushima, S. (1982). Mechanism of signal peptide cleavage in the biosynthesis of the major lipoprotein of the Escherichia coli outer membrane. J Biol Chem 257, 5177-5182.

Innis, M. A., Tokunaga, M., Williams, M. E., Loranger, J. M., Chang, S. Y., Chang, S. \& Wu, H. C. (1984). Nucleotide sequence of the Escherichia coli prolipoprotein signal peptidase (lsp) gene. Proc Natl Acad Sci U S A 81, 3708-3712.

Inukai, M., Ghrayeb, J., Nakamura, K. \& Inouye, M. (1984). Apolipoprotein, an intermediate in the processing of the major lipoprotein of the Escherichia coli outer membrane. J Biol Chem 259, 757-760.

Janulczyk, R., Ricci, S. \& Bjorck, L. (2003). MtsABC is important for manganese and iron transport, oxidative stress resistance, and virulence of Streptococcus pyogenes. Infect Immun 71, 2656-2664.

Jones, C. L., Monaghan, P., Field, T. R., Smith, A. J., Ward, P. N. \& Leigh, J. A. (2004). Localization of MtuA, an LraI homologue in Streptococcus uberis. J Appl Microbiol 97, 149-157.

Kontinen, V. P., Saris, P. \& Sarvas, M. (1991). A gene (prsA) of Bacillus subtilis involved in a novel, late stage of protein export. $\mathrm{Mol}$ Microbiol 5, 1273-1283.

Lee, N., Yamagata, H. \& Inouye, M. (1983). Inhibition of secretion of a mutant lipoprotein across the cytoplasmic membrane by the wildtype lipoprotein of the Escherichia coli outer membrane. J Bacteriol 155, 407-411.

Leskela, S., Wahlstrom, E., Kontinen, V. P. \& Sarvas, M. (1999). Lipid modification of prelipoproteins is dispensable for growth but essential for efficient protein secretion in Bacillus subtilis: characterization of the Lgt gene. Mol Microbiol 31, 1075-1085.

Machata, S., Tchatalbachev, S., Mohamed, W., Jansch, L., Hain, T. \& Chakraborty, T. (2008). Lipoproteins of Listeria monocytogenes are critical for virulence and TLR2-mediated immune activation. $J$ Immunol 181, 2028-2035.

Maguin, E., Prevost, H., Ehrlich, S. D. \& Gruss, A. (1996). Efficient insertional mutagenesis in lactococci and other gram-positive bacteria. J Bacteriol 178, 931-935.

Petit, C. M., Brown, J. R., Ingraham, K., Bryant, A. P. \& Holmes, D. J. (2001). Lipid modification of prelipoproteins is dispensable for growth in vitro but essential for virulence in Streptococcus pneumoniae. FEMS Microbiol Lett 200, 229-233.

Qi, H. Y., Sankaran, K., Gan, K. \& Wu, H. C. (1995). Structure-function relationship of bacterial prolipoprotein diacylglyceryl transferase: functionally significant conserved regions. J Bacteriol 177, 6820-6824.

Reizer, J., Hoischen, C., Titgemeyer, F., Rivolta, C., Rabus, R., Stulke, J., Karamata, D., Saier, M. H., Jr \& Hillen, W. (1998). A novel protein kinase that controls carbon catabolite repression in bacteria. Mol Microbiol 27, 1157-1169.

Sankaran, K. \& Wu, H. C. (1994). Lipid modification of bacterial prolipoprotein. Transfer of diacylglyceryl moiety from phosphatidylglycerol. J Biol Chem 269, 19701-19706.
Sibbald, M. J., Ziebandt, A. K., Engelmann, S., Hecker, M., de Jong, A., Harmsen, H. J., Raangs, G. C., Stokroos, I., Arends, J. P. \& other authors (2006). Mapping the pathways to staphylococcal pathogenesis by comparative secretomics. Microbiol Mol Biol Rev 70, 755-788.

Smith, A. J., Ward, P. N., Field, T. R., Jones, C. L., Lincoln, R. A. \& Leigh, J. A. (2003). MtuA, a lipoprotein receptor antigen from Streptococcus uberis, is responsible for acquisition of manganese during growth in milk and is essential for infection of the lactating bovine mammary gland. Infect Immun 71, 4842-4849.

Stoll, H., Dengjel, J., Nerz, C. \& Gotz, F. (2005). Staphylococcus aureus deficient in lipidation of prelipoproteins is attenuated in growth and immune activation. Infect Immun 73, 2411-2423.

Sutcliffe, I. C. \& Harrington, D. J. (2002). Pattern searches for the identification of putative lipoprotein genes in Gram-positive bacterial genomes. Microbiology 148, 2065-2077.

Sutcliffe, I. C. \& Harrington, D. J. (2004). Lipoproteins of Mycobacterium tuberculosis: an abundant and functionally diverse class of cell envelope components. FEMS Microbiol Rev 28, 645-659.

Sutcliffe, I. C. \& Russell, R. R. (1995). Lipoproteins of gram-positive bacteria. J Bacteriol 177, 1123-1128.

Tjalsma, H., Kontinen, V. P., Pragai, Z., Wu, H., Meima, R., Venema, G., Bron, S., Sarvas, M. \& van Dijl, J. M. (1999a). The role of lipoprotein processing by signal peptidase II in the Gram-positive eubacterium Bacillus subtilis. Signal peptidase II is required for the efficient secretion of alpha-amylase, a non-lipoprotein. J Biol Chem 274, 1698-1707.

Tjalsma, H., Zanen, G., Venema, G., Bron, S. \& van Dijl, J. M. (1999b). The potential active site of the lipoprotein-specific (type II) signal peptidase of Bacillus subtilis. J Biol Chem 274, 28191-28197.

Tokunaga, H. \& Wu, H. C. (1984). Studies on the modification and processing of prolipoprotein in Escherichia coli. Effects of structural alterations in prolipoprotein on its maturation in wild type and lpp mutants. J Biol Chem 259, 6098-6104.

Tokunaga, M., Tokunaga, H. \& Wu, H. C. (1982). Post-translational modification and processing of Escherichia coli prolipoprotein in vitro. Proc Natl Acad Sci U S A 79, 2255-2259.

Tokunaga, M., Loranger, J. M., Chang, S. Y., Regue, M., Chang, S. \& Wu, H. C. (1985). Identification of prolipoprotein signal peptidase and genomic organization of the lsp gene in Escherichia coli. J Biol Chem 260, 5610-5615.

von Heijne, G. (1989). The structure of signal peptides from bacterial lipoproteins. Protein Eng 2, 531-534.

Wahlstrom, E., Vitikainen, M., Kontinen, V. P. \& Sarvas, M. (2003). The extracytoplasmic folding factor PrsA is required for protein secretion only in the presence of the cell wall in Bacillus subtilis. Microbiology 149, 569-577.

Ward, P. N., Field, T. R., Ditcham, W. G., Maguin, E. \& Leigh, J. A. (2001). Identification and disruption of two discrete loci encoding hyaluronic acid capsule biosynthesis genes has $A$, has $B$, and has $C$ in Streptococcus uberis. Infect Immun 69, 392-399.

Yamagata, H. (1983). Temperature-sensitive prolipoprotein signal peptidase in an Escherichia coli mutant: use of the mutant for an efficient and convenient assay system. J Biochem 93, 1509-1515.

Yamagata, H., Ippolito, C., Inukai, M. \& Inouye, M. (1982). Temperature-sensitive processing of outer membrane lipoprotein in an Escherichia coli mutant. J Bacteriol 152, 1163-1168.

Yamaguchi, K., Yu, F. \& Inouye, M. (1988). A single amino acid determinant of the membrane localization of lipoproteins in E. coli. Cell 53, 423-432.

Edited by: T. Palmer 